ISSN: 1021-7444

\title{
CARACTERIZACIÓN MOLECULAR Y GERMINACIÓN DE SEMILLAS DE MAÍCES CRIOLLOS AZULES CON ENVEJECIMIENTO ACELERADO $^{1}$
}

\author{
Dagoberto Durán-Hernández ${ }^{2}$, Germán Fernando Gutiérrez-Hernández², José Luis Arellano-Vázquez, \\ Elpidio García-Ramírez $z^{4}$ Juan Virgen-Vargas ${ }^{3}$
}

\section{RESUMEN}

Caracterización molecular y germinación de semillas de maíces criollos azules con envejecimiento acelerado. El objetivo del presente estudio fue determinar el efecto del envejecimiento acelerado en semillas de variedades criollas de maíz azul. En los laboratorios de la Universidad Nacional Autónoma de México y del Instituto Politécnico Nacional, semillas de cuatro variedades criollas de maíz azul (Cuijingo, Cocotitlán, Puebla y Oaxaca), se sometieron a dos tipos de envejecimiento acelerado: calor húmedo $\left(41^{\circ} \mathrm{C}, 72\right.$ horas y $100 \%$ h. r.) y calor seco $\left(60^{\circ} \mathrm{C}, 48\right.$ horas). Se evaluaron: protrusión radicular de 0 a 72 horas de imbibición y, a los siete días de incubación a $25^{\circ} \mathrm{C}$, plántulas normales, anormales y semillas muertas. Se obtuvo la caracterización molecular de los tratamientos mediante la RAPD y se construyeron los dendogramas respectivos. En plántulas normales provenientes de semillas sin envejecimiento acelerado, las variedades Oaxaca y Puebla fueron significativamente superiores. El peso seco en las plántulas disminuyó con el deterioro, siendo exigua con calor seco. La presencia de plántulas anormales se incrementó con ambos tipos de deterioro en el orden: Oaxaca, Puebla, Cocotitlán y Cuijingo. La diferenciación de los perfiles de bandeo entre semillas testigo y deterioradas ocurrió con la secuencia: Oaxaca (31,6\%), Cocotitlán (25,8\%), Puebla (19,6\%) y Cuijingo (18,6\%). Estos valores de similitud, a los cuales se ramificaron los perfiles genómicos, se asociaron de manera inversa con el nivel de vigor de las semillas, y directamente con la presencia de anormalidades en las plántulas obtenidas.

Palabras clave: Zea mays, viabilidad seminal, huella genómica, RAPDs.

\begin{abstract}
Molecular characterization and seed germination of blue corn landraces with accelerated aging. The objective of this work was to determine the effect of accelerated aging on seeds of blue corn landraces. The study was conducted in the laboratories of National Polytechnic Institute and National University of México. Seeds of four blue corn landraces (Cuijingo, Cocotitlán, Puebla and Oaxaca), were subjected to accelerated aging treatments: humid heat $\left(\mathrm{HH}, 41^{\circ} \mathrm{C}, 72\right.$ $\mathrm{h}$, and $100 \% \mathrm{r}$. h.), and dry heat $\left(\mathrm{DH}, 60^{\circ} \mathrm{C}, 48 \mathrm{~h}\right.$. The parameters measured were: radicle protrusion (RP) in at 0 to 72 $\mathrm{h}$ of imbibition, and after seven days of incubation at $25^{\circ} \mathrm{C}$, normal seedlings (NS), abnormal seedlings (AS), and dead seeds (DS) were counted. The molecular characterization by RAPD was also performed and the respective dendrograms were constructed. In normal seedlings obtained from seeds without accelerated ageing, the varieties Oaxaca and Puebla were significantly superior. Seedling dry weight diminished with deterioration, being minimum under dry heat. The number of abnormal seedlings increased under both deterioration treatments, in the order Oaxaca, Puebla, Cocotitlán and Cuijingo. Differentiation of the band profiles among control and deteriorated seeds occurred in the sequence: Oaxaca (31,6\%), Cocotitlán $(25,8 \%)$, Puebla $(19,6 \%)$ and Cuijingo $(18,6 \%)$. These similarity values were inversely related to seed vigour, and irectly related to the presence of abnormalities in seedlings.
\end{abstract}

Key words: Zea mays, seed viability, genomic finger printing, RAPDs.

1 Recibido: 18 de abril, 2010. Aceptado: 16 de mayo, 2011. Parte de la Tesis de Doctorado en Ciencias del primer autor.

2 Sección de Estudios de Posgrado e Investigación (SEPI) y Departamento de Bioprocesos, Unidad Profesional Interdisciplinaria de Biotecnología (UPIBI), Instituto Politécnico Nacional (IPN). Av. Acueducto s/n. C. P. 07340, La Laguna Ticoman. México, D. F. Tel. (01-55) 5729-6000, ext. 56395. duranhd78@yahoo.com.mx; enredipn@yahoo.com.mx (autor para correspondencia).

3 Programa de Maíz, Campo Experimental Valle de México (CEVAMEX), Instituto Nacional de Investigaciones Forestales, Agrícolas y Pecuarias (INIFAP). Apartado Postal 10.56230, Chapingo, México. arevajolu@yahoo.com.mx

4 Facultad de Química, Universidad Nacional Autónoma de México (UNAM). Av. Universidad y Copilco, Ciudad Universitaria. C. P. 04510, México, D. F. elpidio@servidor.unam.mx 


\section{INTRODUCCIÓN}

En México se preserva actualmente el cultivo de maíces criollos porque satisfacen necesidades específicas de la población por su adaptación, sabor o color. Además, constituyen la base para la actividad agrícola tradicional y son una fuente de variabilidad para el fitomejoramiento de este primordial cultivo, de modo que es prioritario su conocimiento (Hernández y Esquivel 2004).

En los Valles Altos Centrales de México (Puebla, Tlaxcala, México e Hidalgo) se cultivan alrededor de 1,5 millones de hectáreas de maíz, de las cuales el $85 \%$ es de temporal y el 35\% de los productores siembra maíz criollo pigmentado (azul principalmente), del que se estima una cosecha anual de 300 mil toneladas (Antonio et al. 2004). Los distintos colores del maíz pigmentado (negro, morado, azul o rojo) se atribuyen a la presencia de antocianinas en la capa de aleurona y/o en el pericarpio de la semilla (Salinas et al. 1999). En particular, la antocianina azul se encuentra en la capa de aleurona (Beltrán et al. 2001). Las antocianinas se derivan de la cianidina y la pelargonidina, los cuales son compuestos fenólicos del grupo de los flavonoides (Harborne y Gavazzi 1969). Son cromóforos solubles en agua y están relacionados con la reducción de colesterol y triglicéridos en el torrente sanguíneo humano, por lo que se recomienda su ingesta para prevenir o tratar afecciones cardiacas.

De las antocianinas del maíz se obtienen colorantes de vinos, mermeladas y jugos de fruta (Maltros et al. 1999). Los flavonoides también presentes en el maíz azul son antioxidantes (Dickerson 1990). Además, el maíz azul con endospermo harinoso contiene mayor contenido de proteínas, minerales y aminoácidos esenciales que el blanco o amarillo (Dickerson 1990). El maíz azul posee un exótico y atractivo color, lo cual se suma a su mejor sabor (Antonio et al. 2004), y por estos atributos sensoriales se ha elevado su demanda en el mercado y alcanza mejores precios que el maíz amarillo o incluso blanco (Durán 2008).

A pesar de la existencia de numerosas variedades criollas de maíz azul y ser muy apreciado por los productores de autoconsumo y recientemente por consumidores urbanos, esta variante adolece de serias limitantes para su producción y almacenamiento, ya que presenta alta susceptibilidad a las plagas de almacén y rápida pérdida de vigor y viabilidad de sus semillas
(Durán 2008), además de carecer de una descripción varietal precisa.

Por lo anterior, en el presente estudio se analizó la existencia de variación genética para longevidad de semilla en maíces criollos azules, se utilizó el sistema de envejecimiento artificial o acelerado (Fragoso et al. 2006), en el cual se emplean calor y/o humedad para inducir daños similares a los ocurridos en las semillas durante su almacenamiento.

Son diversas las alteraciones causadas por el envejecimiento, considerándose entre las principales a las ocurridas en la estructura y funcionalidad del ADN (McDonald 1999), las cuales eventualmente pueden ser reparadas durante la imbibición seminal (Elder y Osborne 1993) con una eficiencia dependiente del genotipo y de la intensidad de los daños (Gutiérrez et al. 1993). Fisiológicamente estas anomalías se expresan en germinación lenta, plántulas malformadas, escasez de biomasa y pérdida de vigor.

Por otra parte, la identificación de las variedades de cultivo tradicionalmente se ha basado en aspectos morfológicos (Zhang et al. 1996), los cuales en su mayoría son caracteres continuos, lo cual significa que están presentes en todas las variedades sólo que fluctúa su nivel de expresión, mismo que es afectado por el ambiente (Cooke 1999). Estudios señalan las limitaciones intrínsecas de las metodologías convencionales de caracterización varietal (Gutiérrez et al. 2009). También se ha señalado que las técnicas moleculares de identificación genotípica no interaccionan con el ambiente, reconocen directamente las diferencias genéticas entre individuos a nivel del ADN y se obtiene un perfil genómico específico para cada variedad (Morell et al. 1995, Zhang et al. 1996, Marcos-Filho y McDonald 1998).

En este trabajo también se abordó la caracterización molecular de los maíces azules y se empleó la amplificación aleatoria del ADN polimórfico (RAPD, en inglés), la cual ha sido documentada como un procedimiento confiable para la determinación de la identidad genética de las especies de cultivo (McDonald et al. 1994), y en particular para maíz (Zúñiga 2007, Durán 2008, Gutiérrez et al. 2009).

La metodología de la RAPD fue propuesta por Williams et al. (1990), quienes sugieren utilizar iniciadores de diez nucleótidos de longitud y con una composición de guanina y citosina mayor del $50 \%$, los cuales se alinean con el ADN genómico y se aparean 
las bases complementarias; mediante variaciones cíclicas de temperatura, se sintetizan bandas o amplicones de 200 a 2000 pb por la acción de una enzima ADN polimerasa termoestable (Williams et al. 1990, KleinLankhorst et al. 1991, McDonald et al. 1994).

Los fragmentos amplificados se despliegan según su peso molecular durante la electroforesis en agarosa o poliacrilamida, algunos de ellos pueden ser genotípicoespecíficos y por tanto útiles para determinar la identidad varietal (Zhang et al. 1996), así como para el fitomejoramiento asistido por marcadores moleculares.

Los polimorfismos entre genotipos se manifiestan por la presencia o ausencia de una o más bandas por lo que se consideran dominantes (Williams et al. 1990, Penner 1996), de manera que no es posible distinguir si se trata de un locus heterocigótico u homocigótico (Valadez y Kahl 1999).

Las bandas diferenciales entre genotipos se originan por el cambio de un par de bases, por rupturas y fragmentaciones estructurales, por deleciones que modifican o eliminan el sitio de inserción del iniciador, o por translocaciones que separan los sitios de acoplamiento del iniciador a una distancia mayor a 2500 nucleótidos, a la cual ya no hay amplificación (Xena 2000).

Las señaladas variaciones estructurales en el ADN ocurren durante la evolución de la especie o son causadas por el envejecimiento de las semillas, inclusive se ha reportado la existencia de segmentos del ADN con mayor susceptibilidad a la degradación (Marcos-Filho y McDonald 1998).

El objetivo del presente estudio fue determinar el efecto del envejecimiento acelerado sobre la germinación y el vigor de semillas de variedades criollas de maíz azul.

\section{MATERIALES Y MÉTODOS}

El estudio aquí descrito se efectuó durante el 2008 en el Laboratorio de Biotecnología Molecular de la Unidad Profesional Interdisciplinaria de Biotecnología (UPIBI) del Instituto Politécnico Nacional (IPN) y en la Facultad de Química de la Universidad Nacional Autónoma de México (UNAM).

Se utilizaron semillas de cuatro variedades criollas de maíz con aleurona azul (Cuijingo, Cocotitlán, Puebla y Oaxaca), proporcionadas por el Programa de Maíz del Campo Experimental Valle de México perteneciente al Instituto Nacional de Investigaciones Forestales, Agrícolas y Pecuarias, localizado en Chapingo, México.

\section{Tratamientos}

Las semillas se sometieron a dos modalidades de envejecimiento artificial: calor húmedo $\left(\mathrm{CH}, 41^{\circ} \mathrm{C}\right.$ y $100 \%$ h. r., $72 \mathrm{~h}$ ) y calor seco $\left(\mathrm{CS}, 60^{\circ} \mathrm{C}, 48 \mathrm{~h}\right)$, para cada variedad se incluyó su correspondiente testigo (semillas recién cosechadas sin envejecimiento artificial), de modo que se generaron doce tratamientos.

\section{Protrusión radicular}

Para determinar la protrusión radicular (PR), se colocaron las semillas con el embrión hacia abajo en cajas Petri con papel filtro y $10 \mathrm{ml}$ de agua bidestilada, se incubaron a $25^{\circ} \mathrm{C}$ y cada 24 horas se hizo el conteo hasta que ya no ocurrieron brotaciones ( 72 horas). El criterio para considerar que la radícula había protruido fue que tuviera una longitud mínima de $3 \mathrm{~mm}$ (Fragoso et al. 2006).

\section{Prueba de germinación normal}

Se realizó la prueba de germinación normal o estándar (ISTA 1995). Las semillas se colocaron sobre toallas de papel húmedo las cuales se enrollaron y se colocaron verticalmente dentro de bolsas de plástico a $25 \pm 4^{\circ} \mathrm{C}$. A los siete días se evaluó el número de plántulas normales (PN), y anormales (PA) y la presencia de semillas muertas (SM). Además, se cuantificó el peso seco acumulado en la plúmula (PSP), la radícula (PSR) y el total (PST).

\section{Extracción de ADN genómico}

Se aisló el ADN de cada tratamiento (Dellaporta et al. 1983) a partir de $0,1 \mathrm{~g}$ de embriones de las semillas, los cuales previamente se lavaron dos veces con agua bidestilada y se secaron superficialmente con papel absorbente. Después se cuantificó el ADN y se le determinó la calidad mediante electroforesis en agarosa $(0,7 \%$ p/v) (CIMMYT 2006). 


\section{Amplificación aleatoria del ADN polimórfico (RAPD)}

Selección de iniciadores

Se emplearon diez iniciadores (Cuadro 1), la selección se hizo con base en las características de las bandas amplificadas, tales como nitidez y consistencia (Valadez y Kahl 1999).

Cuadro 1. Iniciadores RAPD y sus secuencias nucleotídicas Facultad de Química, Universidad Nacional Autónoma de México, México. 2008.

\begin{tabular}{cc}
\hline Iniciador & Secuencia $\left(\mathbf{5}^{\prime} \mathbf{3}^{\prime}\right.$ ) \\
\hline RAPD E-08 & CCCAAGGTCC \\
RAPD E-09 & GGTGCGGGAA \\
RAPD E-10 & GAGTCTCAGG \\
RAPD E-11 & TTATCGCCCC \\
RAPD E-12 & TCAGGGAGGT \\
RAPD E-13 & AAGACCCTCC \\
RAPD E-14 & ACGCACAACC \\
RAPD E-15 & GCTGACTGTG \\
RAPD E-16 & CTTCACCCGA \\
RAPD E-17 & CACCAGGCTA \\
RAPD G-01 & CTACGGAGGA \\
RAPD G-03 & GAGCCCTCCA \\
RAPD G-15 & ACTGGGACTC \\
\hline
\end{tabular}

La amplificación (Cuadro 2) se llevó a cabo en un volumen final de $25 \mu \mathrm{l}$ y los fragmentos producidos se separaron mediante electroforesis horizontal en gel de agarosa $(1,2 \% \mathrm{p} / \mathrm{v})$ y se tiñeron con bromuro de etidio (CIMMYT 2006).

\section{Análisis de datos genómicos}

Se codificaron los fragmentos amplificados para cada iniciador, empleando el número uno para indicar la presencia de la banda y el cero para denotar su ausencia. Con su codificación se construyó la matriz base de datos, misma que constó de 63 filas y trece columnas, en correspondencia con las bandas obtenidas y los genotipos estudiados, esto es, los doce tratamientos
Cuadro 2. Programa para la amplificación aleatoria del ADN polimórfico (RAPD). Facultad de Química, Universidad Nacional Autónoma de México. 2008.

\begin{tabular}{ccc}
\hline Ciclos & Temperatura $\left({ }^{\circ} \mathbf{C}\right)$ & Tiempo \\
\hline 1 & 94 & $1^{\prime}$ \\
& & \\
& 94 & $30^{\prime \prime}$ \\
55 & 35 & $30^{\prime \prime}$ \\
& 72 & $1^{\prime} 30^{\prime \prime}$ \\
& & \\
1 & 72 & $2^{\prime} 30^{\prime \prime}$ \\
& 4 & $\alpha$ \\
\hline
\end{tabular}

bajo estudio más el ADN de levadura (Saccharomyces cerevisiae), utilizado como control negativo.

\section{Análisis estadístico}

Los experimentos fisiológicos se manejaron con un diseño de bloques al azar con cuatro repeticiones de veinticinco semillas cada una. Se calcularon los errores estándar $(n=4)$ de protusión radicular, plántulas normales y anormales, semillas muertas y peso seco, y se muestran como barras en las figuras correspondientes (Reyes 1980).

Para construir los dendogramas se utilizó el programa NTSYSpc (Numerical Taxonomy and Multivariate Analysis System, ver. 2.1) (Rohlf 2000), con los parámetros: distancia de Seuil $=0$, confiabilidad del 95\%, el estimador de distancias de Nei y Li, el método de agrupamiento del promedio y el coeficiente de similitud de Jaccard (Dubreuil et al. 2003).

\section{RESULTADOS Y DISCUSIÓN}

\section{Análisis fisiológico}

En las semillas sometidas a envejecimiento o deterioro artificial se advirtió una sensible reducción de la PR en relación a los controles respectivos, es decir, a semillas del mismo genotipo pero recién cosechadas, esta disminución fue mayor en el caso de $\mathrm{CH}$ (Figura 1). En PR, las variedades se desempeñaron de modo 


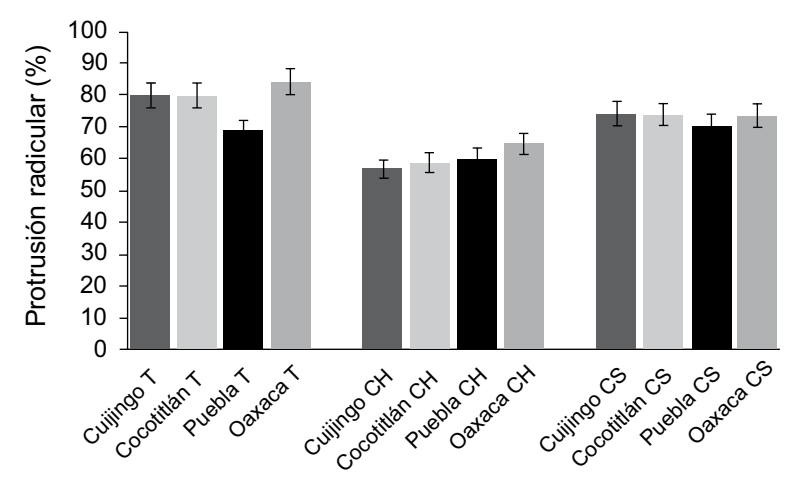

Variedades de maíz azul

Figura 1. Protrusión radicular de las semillas de cuatro variedades de maíz azul, bajo dos tratamientos de envejecimiento artificial. $\mathrm{T}=$ Testigos, $\mathrm{CH}=\mathrm{Calor}$ húmedo $\left(41^{\circ} \mathrm{C}, 72 \mathrm{~h}\right.$ y $100 \%$ h. r. $)$ y $\mathrm{CS}=$ Calor seco $\left(60^{\circ} \mathrm{C}, 48 \mathrm{~h}\right)$. Unidad Profesional Interdisciplinaria de Biotecnología (UPIBI) del Instituto Politécnico Nacional (IPN), México. 2008.

similar dentro de cada modalidad de deterioro $(\mathrm{CH}$ o CS); no obstante, en las evaluaciones hechas a tiempos más avanzados de germinación y que corresponden a la formación de plántulas (siete días de incubación), se expresaron ya diferencias genotípicas significativas entre ellas (Figuras 2, 3 y 4).

Esto indicaría que aunque la brotación radicular es un suceso físico promovido por la rehidratación

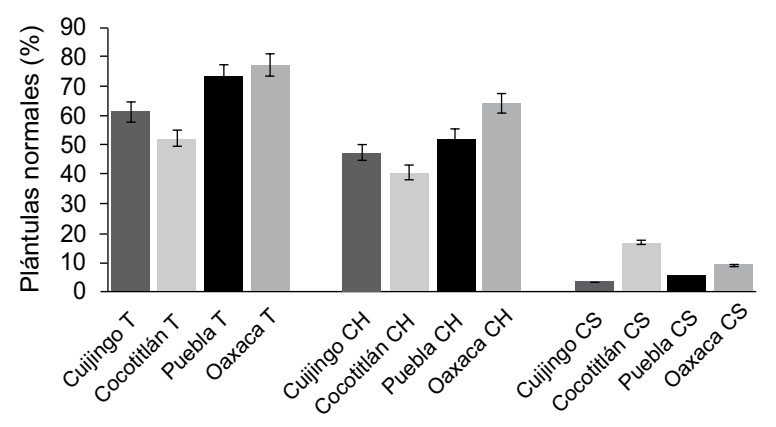

Variedades de maíz azul

Figura 2. Plántulas normales obtenidas en la germinación de las semillas de cuatro variedades de maíz azul, bajo dos tratamientos de envejecimiento artificial. $\mathrm{T}=$ Testigos, $\mathrm{CH}=$ Calor Húmedo $\left(41^{\circ} \mathrm{C}, 72 \mathrm{~h} \mathrm{y}\right.$ $100 \%$ h. r. $)$ y CS $=$ Calor $\operatorname{Seco}\left(60^{\circ} \mathrm{C}, 48\right.$ h $)$. Unidad Profesional Interdisciplinaria de Biotecnología (UPIBI) del Instituto Politécnico Nacional (IPN), México. 2008.

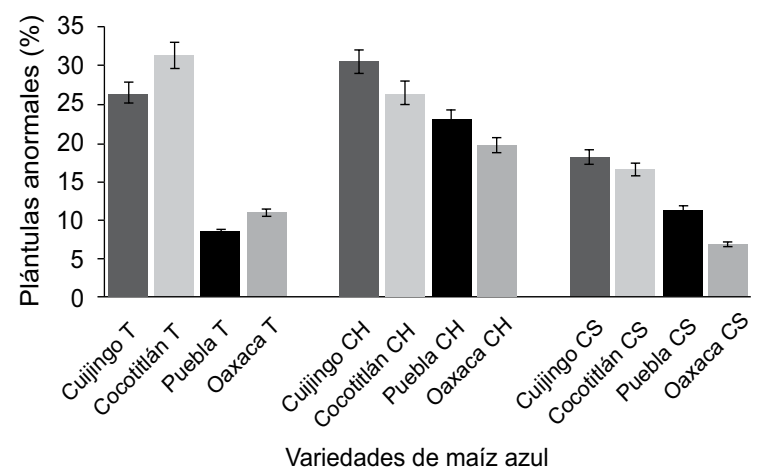

Figura 3. Plántulas anormales obtenidas en germinación de las semillas de cuatro variedades de maíz azul, bajo dos tratamientos de envejecimiento articial. $\mathrm{T}=$ Testigos, $\mathrm{CH}=$ Calor Húmedo $\left(41^{\circ} \mathrm{C}, 72 \mathrm{~h} \mathrm{y}\right.$ $100 \%$ h. r.) y CS $=$ Calor $\operatorname{Seco}\left(60^{\circ} \mathrm{C}, 48 \mathrm{~h}\right)$. Unidad Profesional Interdisciplinaria de Biotecnología (UPIBI) del Instituto Politécnico Nacional (IPN), México. 2008.

de los tejidos seminales (Bewley y Black 1994), se deriva de procesos previos como refuncionalización de membranas, síntesis de macromoléculas, división celular, etc. (Cruz et al. 1995), los cuales se dañaron a consecuencia del envejecimiento, y la expresión fisiológica de tales daños se manifestó hasta la formación de plántulas y con un evidente efecto genotípico.

Es interesante advertir que los daños infligidos por cada tipo de envejecimiento fueron distintos en el

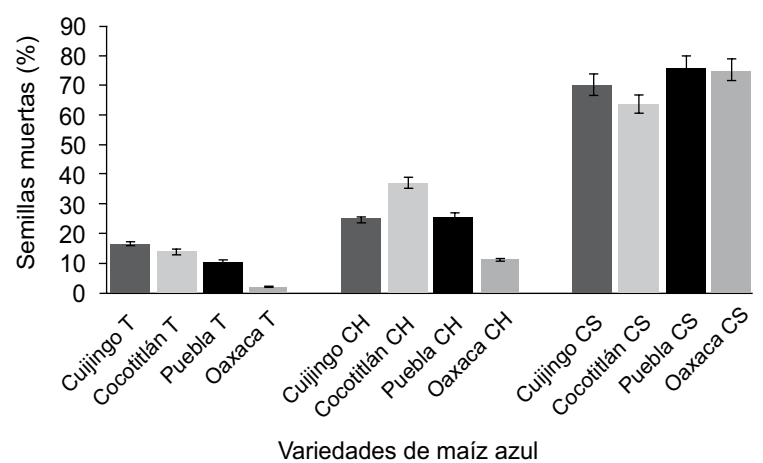

Figura 4. Semillas muertas detectadas en la germinación de cuatro variedades de maíz azul, bajo dos tratamientos de envejecimiento artificial. T=Testigos, $\mathrm{CH}=$ Calor húmedo $\left(41^{\circ} \mathrm{C}, 72 \mathrm{~h}\right.$ y $100 \%$ h. r. $)$ y $\mathrm{CS}=$ Calor seco $\left(60^{\circ} \mathrm{C}, 48 \mathrm{~h}\right)$. Unidad Profesional Interdisciplinaria de Biotecnología (UPIBI) del Instituto Politécnico Nacional (IPN), México. 2008. 
proceso fisiológico que afectaron, en el tiempo en que se expresaron y en la posibilidad de subsanarlos, ya que la mayor disminución de la PR con respecto a los testigos y la mayor proporción de PA, se produjeron en las semillas sometidas a $\mathrm{CH}$, mientras que con $\mathrm{CS}$, las variables de PN, PST y SM presentaron los efectos más adversos, y estas últimas son componentes fundamentales de la germinación por tratarse de las plantas potencialmente productivas, de la capacidad para crecer, desarrollarse y de la muerte celular, respectivamente.

A consecuencia de los tratamientos de envejecimiento, ocurrió una sensible reducción en la cantidad de las PN generadas en los tratamientos de deterioro con respecto a los controles (Figura 2), particularmente con calor seco en el cual sobresale Cocotitlán por mostrar significativamente la mayor tolerancia. Por otra parte, se incrementó la formación de PA (Figura 3) en todas la variedades, a excepción de Cocotitlán genotipo que denotó la mayor tolerancia al calor húmedo en esta medición.

Para ambos tipos de envejecimiento, las PA aumentaron en el orden: Oaxaca, Puebla, Cocotitlán y Cuijingo, mismo que correspondería a su aptitud genotípica decreciente para amortiguar los daños genómicos causados por el envejecimiento, de donde se deriva la longevidad seminal.

Los valores alcanzados para semillas duras o muertas, en las semillas testigo fueron menores al $18 \%$ (Figura 4), pero se incrementaron a consecuencia del envejecimiento, entre 12 y $38 \%$ para calor húmedo y entre 65 y $78 \%$ para calor seco, siendo evidente otra vez la severidad de este último.

De igual modo, la acumulación de peso seco en las plántulas se redujo con el deterioro, siendo casi nula en calor seco (Figura 5). Destacó Oaxaca porque alcanzó el valor más alto entre las semillas control y no perdió esta cualidad con el deterioro (calor húmedo o calor seco), además mostró la mayor cantidad de biomasa tanto en vástago como en radícula; Cuijingo se desempeñó de manera semejante a Oaxaca en $\mathrm{CH}$ sólo que con valores inferiores, mientras que Cocotitlán y Puebla presentaron las menores cantidades. Pese a la escasa materia seca formada en calor seco, sobresalen Oaxaca y Cocotitlán por sus valores superiores, así como Cuijingo que soportó medianamente el $\mathrm{CH}$ pero no el calor seco.

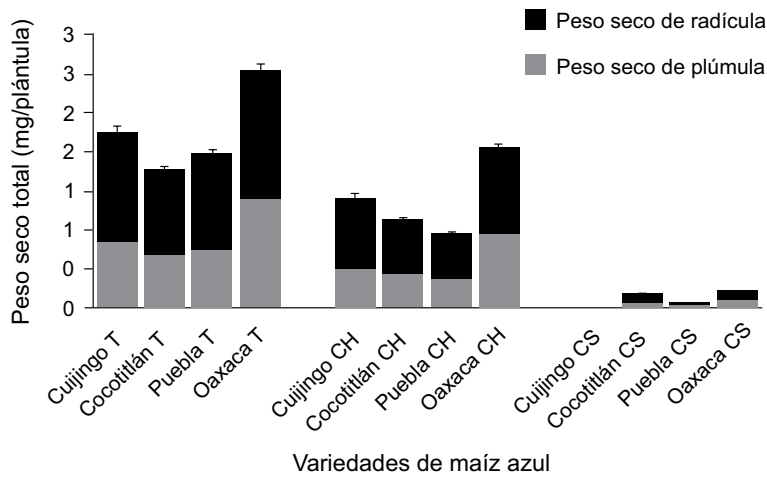

Figura 5. Peso seco de radícula (PSR), de plúmula (PSP) y total (PST) de las plántulas de cuatro variedades de maíz azul, bajo dos tratamientos. T=Testigos, $\mathrm{CH}=$ Calor Húmedo $\left(41^{\circ} \mathrm{C}, 72 \mathrm{~h}\right.$ y $100 \%$ h. r.) y $\mathrm{CS}=$ Calor Seco $\left(60^{\circ} \mathrm{C}, 48 \mathrm{~h}\right)$. Unidad Profesional Interdisciplinaria de Biotecnología (UPIBI) del Instituto Politécnico Nacional (IPN), México. 2008.

\section{Análisis molecular}

\section{Selección de iniciadores RAPD}

En el escrutinio realizado para detectar los iniciadores que amplificaron nítida y claramente segmentos polimórficos de ADN (Figura 6) a la temperatura de alineamiento de $35^{\circ} \mathrm{C}$, óptima para la RAPD (Valadez y Kahl 1999), se escogió al azar un tratamiento (Cuijingo Testigo), el cual se combinó con los iniciadores disponibles, procedimiento que sirvió para elegir diez de ellos (Cuadro 2), con los cuales se desarrolló la RAPD con el templado del ADN extraído de los doce tratamientos más el de Saccharomyces cereviciae.

Se detectaron un total de 63 bandas con longitudes de 370 a 2923 pb, de las cuales sólo dos fueron monomórficas. Los iniciadores E09, E14 y G15 fueron profusamente polimórficos, puesto que en conjunto mostraron el $51 \%$ de la variación total.

La matriz básica de datos consistió de 63 filas (total de bandas) y trece columnas (tratamientos y levadura), con base en ella se calcularon los dendogramas para los tratamientos (Figura 7). Cabe hacer notar que el ADN de levadura sólo mostró una similitud con el maíz del 8 al 11\%, situación lógica dado que se trata de organismos filogenéticamente lejanos y precisamente 

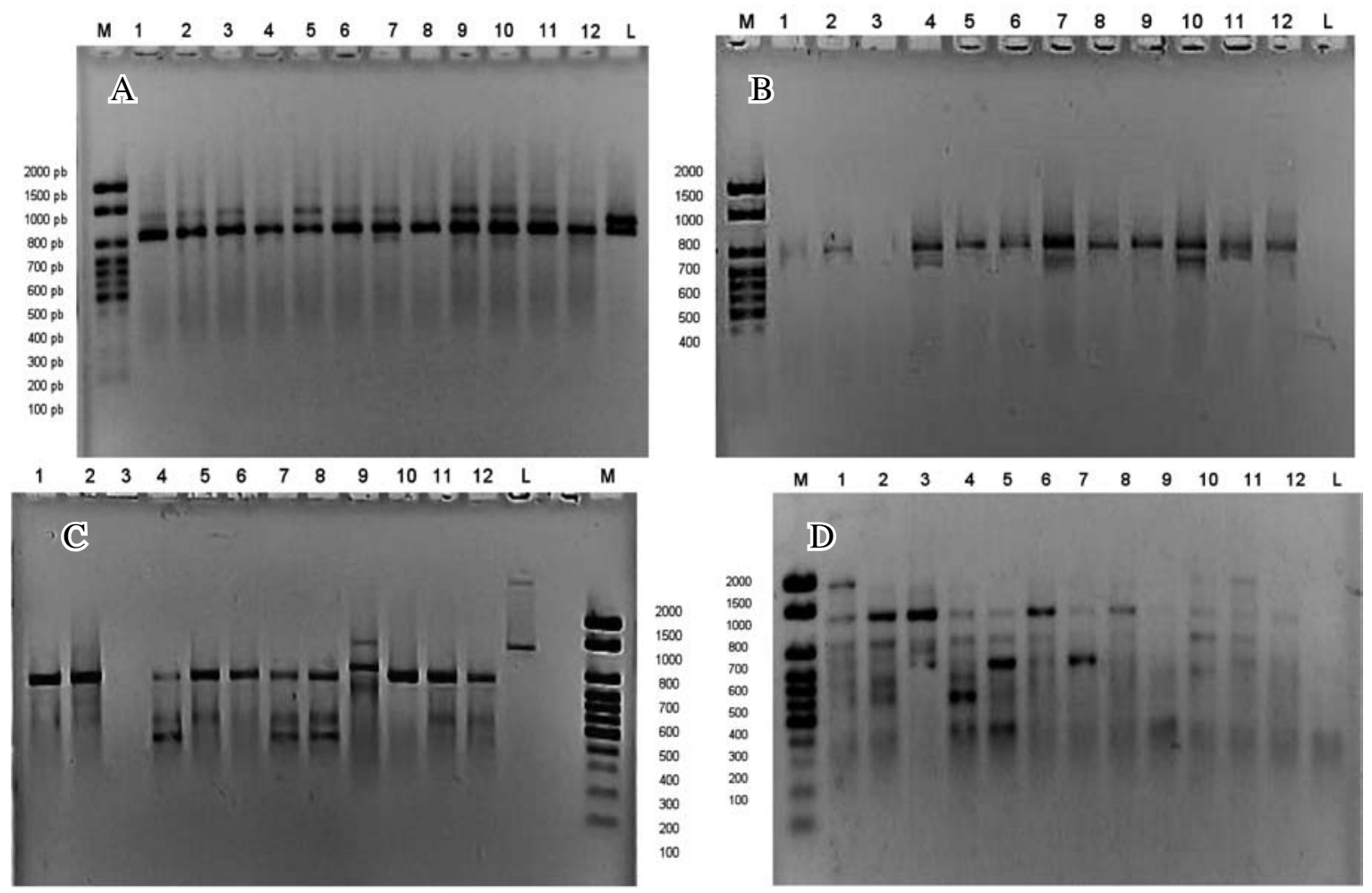

Figura 6. Perfil de bandeo (RAPD) obtenido para semillas de cuatro variedades de maíz azul, bajo dos tratamientos de envejecimiento acelerado. T=Testigos (carriles $1-4), \mathrm{CH}=$ Calor húmedo $\left(41^{\circ} \mathrm{C}, 72 \mathrm{~h}\right.$ y $100 \% \mathrm{~h}$. r.) (carriles 5-8) y $\mathrm{CS}=$ Calor seco $\left(60^{\circ} \mathrm{C}, 48 \mathrm{~h}\right)$ (carriles 9-12) y la levadura (Saccharomyces cereviciae) (L, control negativo), para los iniciadores A) E08, B) E10, C) G13 y D) G15. M=Marcador de peso molecular. pb=pares de bases. Facultad de Química, Universidad Nacional Autónoma de México. 2008.

por ello se empleó como control negativo para constatar la veracidad de los agrupamientos definidos, los cuales tuvieron coeficientes de correlación cofenética con la matriz básica de datos de 0,88 a 0,98 , valores aceptables desde el punto de vista de su reproducibilidad (Gutiérrez et al. 2009).

La formación de conglomerados o grupos de similitud asumió dos niveles jerárquicos, correspondiendo el primero de ellos a la conjunción de los datos genómicos por variedad, y el segundo, a la ponderación intravarietal de las diferencias genómicas causadas por los tratamientos aplicados.

Así, la huella genómica resultó específica para cada variedad independientemente de la condición de las semillas y fue dentro de variedades donde los dendogramas evidenciaron las modificaciones que al nivel del ADN ocurrieron en los embriones durante el envejecimiento artificial. Es decir, los segmentos del ADN testigo, donde por homología de bases se alinearon los iniciadores y hubo amplificación, se alteraron en su estructura molecular a causa del deterioro impuesto (Xena 2000), por lo que fueron diferentes los perfiles genómicos entre uno y otro tipo de semillas (McDonald 1999).

Los perfiles de bandeo de semillas testigo y las sometidas a envejecimiento (calor húmedo y calor seco) fueron semejantes hasta un coeficiente de similitud específico para cada variedad: $31,6 \%$ para Oaxaca, 25,8\% para Cocotitlán, 19,6\% para Puebla y 18,6\% para Cuijingo (Figura 7), a partir del cual los dendogramas se ramificaron y diversificaron los señalados perfiles. Lo anterior denota un efecto genotípico relacionado con la capacidad para amortiguar, tolerar o restaurar los daños infligidos por el envejecimiento artificial sobre el ADN, misma que tendría la mayor expresión en Oaxaca y le seguirían Cocotitlán, Puebla 

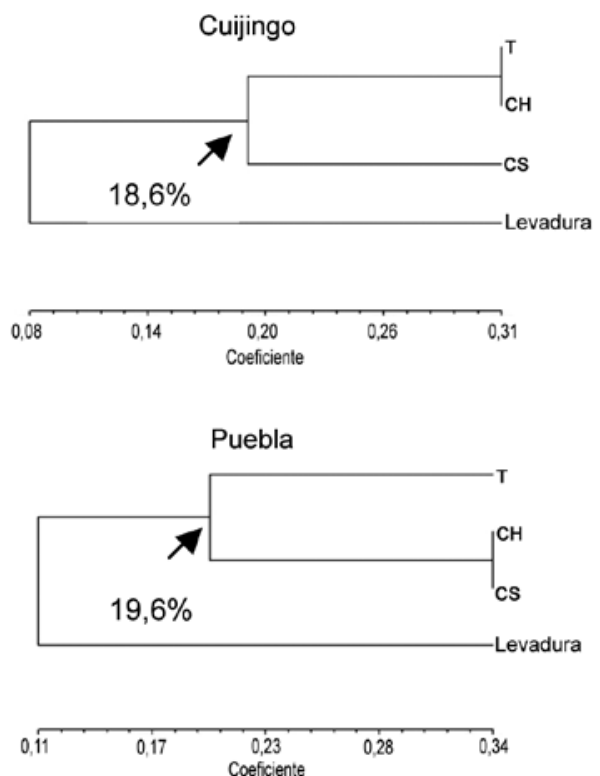
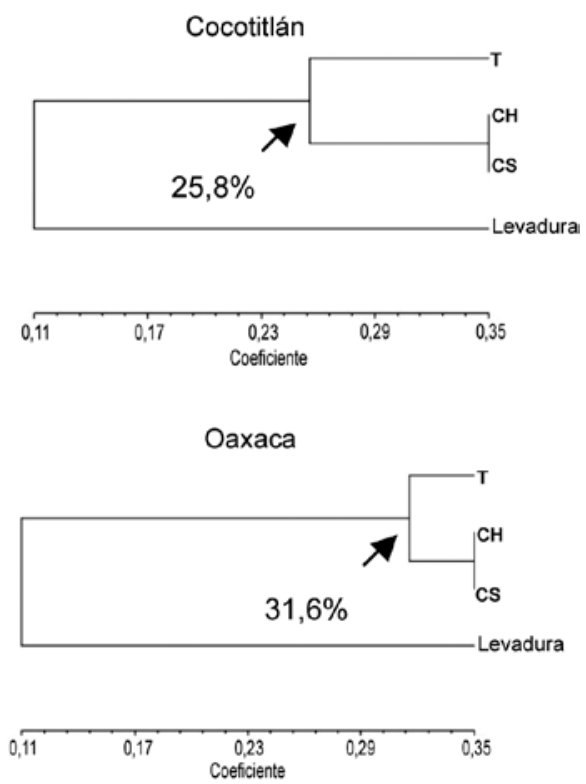

Figura 7. Dendogramas obtenidos con la matriz básica de datos para cada variedad de maíz azul bajo envejecimiento acelerado. T=Testigos, $\mathrm{CH}=$ Calor Húmedo $\left(41^{\circ} \mathrm{C}, 72 \mathrm{~h}\right.$ y $100 \%$ h. r. $)$ y $\mathrm{CS}=\mathrm{Calor}$ Seco $\left(60^{\circ} \mathrm{C}, 48 \mathrm{~h}\right)$. Método de agrupamiento del promedio (UPGMA), basado en el coeficiente de similitud de Jaccard. NTSYSpc. Se indica el coeficiente de similitud (\%) al cual se diversificaron los tratamientos. Facultad de Química, Universidad Nacional Autónoma de México. 2008.

y Cuijingo, de acuerdo a sus coeficientes de similitud descendentes (Figura 7), situación que pudiera relacionarse con la existencia de segmentos de ADN con distinto grado de susceptibilidad al deterioro (MarcosFilho y McDonald 1998).

La secuencia de tolerancia al envejecimiento (Oaxaca $>$ Cocotitlán $>$ Puebla $>$ Cuijingo) detectada a nivel molecular, en varios casos se relacionó con la respuesta fisiológica de las semillas durante su germinación: a) La notoria capacidad de Oaxaca para preservar su ADN pese al envejecimiento, se asoció con un alto desempeño fisiológico, dado que también es la variedad de mayor vigor en semillas testigos y deterioradas (Figuras 2, 3 y 4); b) Cuijingo, genotipo en el cual se mantuvieron agrupadas las semillas testigo con las sometidas a calor húmedo (Figura 7) y cuyas diferencias fisiológicas en las variables plántulas normales, plántulas anormales y MST, aunque significativas estadísticamente, no fueron tan amplias como en las otras variedades (Figuras 2, 3 y 4); y c) La formación de PA (Figura 3) estuvo inversamente vinculada con la resistencia al envejecimiento por calor húmedo y calor seco.

\section{AGRADECIMIENTOS}

Nuestro reconocimiento a las siguientes instituciones: Secretaría de Investigación y Posgrado del IPN, Programa de Estímulos al Desempeño de los Investigadores del IPN, Comisión de Operación y Fomento de Actividades Académicas del IPN, Facultad de Química de la UNAM y al Instituto Nacional de Investigaciones Forestales, Agrícolas y Pecuarias.

\section{LITERATURA CITADA}

Antonio, MM; Arellano, JL; García, G; Miranda, S; Mejía, JA; González, FV. 2004. Variedades criollas de maíz azul raza chalqueño. Características Agronómicas y Calidad de Semillas. Revista Fitotecnia Mexicana 27(1):9-15. 
Beltrán, J; Bockholt, AJ; Rooney, L. 2001. Blue corn. In Hallauer, AR. ed. Specialty Corns. Iowa State University. Ames Iowa, USA. p. 293-337.

Bewley, JD; Black, AM. 1994. Seeds: physiology of development and germination. 2 ed Plenum Press. N. Y. USA. 445 p.

CIMMYT (Centro Internacional de Mejoramiento de Maíz y Trigo). 2006. Protocolos de laboratorio: Laboratorio de Genética Molecular Aplicada del CIMMYT. 3 ed. El Batán Texcoco, Méx. 62 p.

Cooke, RJ. 1999. Modern methods for cultivar verification and transgenic plant challenge. Seed Science and Technology 27:669-680.

Cruz, F; González, VA; Molina J; Vázquez, JM. 1995. Seed deterioration and respiration as related to DNA metabolism in germinating maize seeds. Seed Science and Technology 23:477-486.

Dellaporta, SL; Word, J; Hicks, JB. 1983. A plant DNA minipreparation: Version II. Plant Molecular Biology Rep. 1:19-21.

Dickerson, GW. 1990. Blue corn production and marketing in New Mexico. Coop. Ext. Serv. Guide H-226. p. 294.

Dubreuil, P; Dillman, C; Warburton, M; Crossa, J; Franco, BC. 2003. Users manual for the LCDMV software (calculation software of molecular distance between varieties) for fingerprinting and genetic diversity studies. CIMMYT. El Batán Texcoco, Méx.

Durán, D. 2008. Caracterización fisiológica y molecular de semillas de maíz azul. Tesis de Maestría en Ciencias. UPIBI-IPN. 98 p.

Elder, RH; Osborne, DJ. 1993. Function of DNA synthesis and DNA repair in the survival of embryos during early germination and dormancy. Seed Science Research 3:43-43.

Fragoso, E; Gutiérrez, GF; Virgen, J. 2006. Repercusiones físicas y fisiológicas del envejecimiento natural de semillas de cruzas simples de maíz y sus líneas progenitoras. Revista Fitotecnia Mexicana 29:75-80.

Gutiérrez, GF; Cruz, F; Moreno, J; González, VA; Vázquez, JM. 1993. Natural and artificial seed ageing in maize: germination and DNA synthesis. Seed Science Research 3:279-285.

Gutiérrez, GF; García, E; Arellano, JL; Virgen, J. 2009. Comparación molecular de la identidad genética de genotipos de maíz de diferente edad. Agronomía Mesoamericana 20(1):01-09.

Harborne, JB; Gavazzi, G. 1969. Effect of $\mathrm{Pr}$ and $\mathrm{pr}$ alleles on anthocyanins biosynthesis in Zea mays. Phytochemistry 8:999-1001.
Hernández, JM; Esquivel, G. 2004. Rendimiento de grano y características agronómicas en germoplasma de maíz de valles altos de México. Revista Fitotecnia Méxicana 27:27-31.

ISTA (International Seed Testing Association). 1995. Handbook of vigour test methods. 2 Ed. Zurich, Suiza. $117 \mathrm{p}$.

Klein-Lankhorst, RM; Vermut, A; Weide, R; Liharska, T; Zabel, P. 1991. Isolation of molecular markers for tomato (L. esculentum) using random amplified polymorphic DNA (RAPD). Theor. Appl. Genet. 85:961-968.

Maltros, RH; Ibave, JL; Bustillos, P; Díaz de la G, RI. 1999. Industrialización del maíz como fuente de pigmentos para la industria alimentaria, farmacéutica y de cosméticos. In Espinoza, J; Del Boque, CJ. eds. Memoria Segundo Taller Nacional de Especialidades de Maíz. Saltillo Coahuila, México. Universidad Autónoma Agraria Antonio Narro. p. 35-37.

Marcos-Filho, J; Mcdonald, MB. 1998. Sensitivity of RAPD analysis, germination and vigour test to detect the intensity of deterioration of naturally and artificially aged soybean seeds. Seed Science and Technology 26:141-157.

Mcdonald, MB; Elliot, LJ; Sweeney, PM. 1994. DNA extraction from dry seeds for RAPD analysis in varietal identification studies. Seed Science and Technology 22:171-176.

McDonald, MB. 1999. Seed deterioration: physiology, repair and assessment. Seed Science and Technology 27:177237.

Morell, MK; Peakall, R; Appels, R; Preston, LR; Lloyd, HL. 1995. DNA profiling techniques for plant variety identification. Aust. J. Exp. Agric. 35:807-819.

Penner, GA. 1996. RAPD analysis of plant genomes. Methods of genome analysis in plants 15:251-264.

Reyes, P. 1980. Bioestadística aplicada: Agronomía, Biología, Química. Ed. Trillas, México, D. F. 213 p.

Rohlf, F. J. 2000. NTSYSpc Numerical taxonomy and multivariate analysis system. Version 2.1. Owner's manual. New York Exeter.

Salinas, Y; Soto, M; Martínez, F.; González, V; Ortega, R. 1999. Análisis de antocianinas en maíces de grano azul y rojo provenientes de cuatro razas. Revista Fitotecnia Mexicana 22:161-167.

Valadez, E; Kahl, G. 1999. Categorías básicas para el análisis de marcadores genético-moleculares. Depto. de Fitotecnia, Universidad Autónoma Chapingo, Texcoco, México. 182 p.

AGRONOMÍA MESOAMERICANA 22(1):11-20. 2011 
Williams, JGK; Kubelik, AR; Livak, KJ; Rafalski, JA; Tingey, SV. 1990. DNA polymorphisms amplified by arbitrary primers are useful as genetic markers. Nucleic Acids Research 18:6531-6335.

Xena, EN. 2000. Una década de aplicaciones del método RAPD: Alcances y límites en el estudio de relaciones genéticas en plantas. Acta Científica Venezolana. 51:197-206.
Zhang, J; Mcdonald, MB; Sweeney, PM. 1996. Random amplified polymorphic DNA (RAPDs) from seeds of differing soybean and maize genotypes. Seed Science and Technology 24:513-522.

Zúñiga, F. 2007. Comparación molecular de orígenes de semillas de maíz. Tesis Profesional. Ing. Biotecnólogo. UPIBI, IPN. 51 p. 